https://helda.helsinki.fi

\title{
The Development of Gödel's ontological Proof
}

\section{Kanckos, Annika}

2019-11-15

Kanckos , A \& Lethen , T 2019 , ' The Development of Gödel's ontological Proof ' , The

Review of Symbolic Logic , vol. 14 , no. 4 , pp. 1011-1029 . https://doi.org/10.1017/S1755020319000479

http://hdl.handle.net/10138/323267

https://doi.org/10.1017/S1755020319000479

acceptedVersion

Downloaded from Helda, University of Helsinki institutional repository.

This is an electronic reprint of the original article.

This reprint may differ from the original in pagination and typographic detail.

Please cite the original version. 


\title{
The Development of Gödel's Ontological Proof*
}

\author{
Annika Kanckos ${ }^{\dagger} \&$ Tim Lethen ${ }^{\ddagger}$
}

September 17, 2019

\begin{abstract}
Gödel's ontological proof is by now well-known based on the 1970version, written in Gödel's own hand, and Scott's version of the proof. In this paper new manuscript sources found in Gödel's Nachlass are presented. Three versions of Gödel's ontological proof have been transcribed, and completed from context as true to Gödel's notes as possible. The discussion in this paper is based on these new sources and reveals Gödel's early intentions of a liberal comprehension principle for the higher order modal logic, an explicit use of second order Barcan schemas, as well as seemingly defining a rigidity condition for the system. None of these aspects occur explicitly in the later 1970 version, and therefore they have long been in focus of the debate on Gödel's ontological proof.
\end{abstract}

Keywords: Ontological Argument, Kurt Gödel, Higher-Order Logic (03B15), Modal Logic (03B45).

\section{Introduction}

In the Nachlass of Gödel there are several sketches of the ontological argument, one of which has not been published before, and two versions that were transcribed in the Appendix of the collected works of Gödel [11, Appendix B]. These versions can be added to the known version of Gödel dated Feb 10, 1970, and Scott's version [21] that have been thoroughly researched and even computer analysed and the latter verified (see [4] and related papers). In this paper we will for the first time treat these sketches of the ontological argument as complete formalizations comparable to the known versions; this is made possible partly because of a new transcription of the notes, which has corrected some formulas that were previously misinterpreted, and prove the theorems of these three versions in order to show the development of Gödel's argument.

\footnotetext{
* Author order is alphabetical by surname.

${ }^{\dagger}$ Department of Philosophy, P.O. box 24 (Unioninkatu 40 A), FI - 00014 UNIVERSITY OF HELSINKI, FINLAND. annika.kanckos@helsinki.fi

‡Department of Philosophy, P.O. box 24 (Unioninkatu 40 A), FI - 00014 UNIVERSITY OF HELSINKI, FINLAND. lethen@cs.uni-saarland.de
} 
These three axiomatizations have been transcribed from Gabelsberger shorthand by the second author. The notes occur in the Nachlass as formulas appended with comments in Gabelsberger. Throughout the paper the transcription of Gödel's original notes will be written within brackets. Footnotes will indicate the reference to where in the Nachlass the passage is found. Box and folder numbers refer to the originals in the Princeton archive whereas reel and frame numbers refer to the unique identification of each page in the microfilm version of the archive. The identification follows the inventory Finding Aid of $[12]$.

For the reader's convenience, formal proofs of each version have been collected into an appendix (section 10), which can be read separately from the main paper. Sections $6-8$ of the main paper contain informal proofs of each version of the ontological argument. These informal proofs add as little as possible to Gödel's notes, by not explicitly specifying any formal system for the argument, while still making steps of reasoning clearer. Some potentially questionable properties of the formal system of the appendix are motivated by Gödel's notes, such as using an S5 classical system for a rigid constant domain higher order modal logic. These properties of the intended system of the argument will be discussed in the main paper based on Gödel's notes. Though an axiomatic logic with linear derivations may have been closer to Gödel's style of writing, we will display the formal arguments of the appendix in natural deduction style.

\section{From Anselm of Canterbury to Gödel: the study of the argument}

The ontological proof dates as far back as Anselm of Canterbury (1033-1109) and is generally considered to have reached Gödel's awareness through his interest in Leibniz' philosophy [1, p. 389] and [22, p. 241]. In the Nachlass of Gödel, there is a multitude of scattered notes on theology as well as material in notebooks dedicated to the topic. However, as mentioned in [1, p. 389] little has been found so far in Gödel's notes on Leibniz and the ontological argument.

General information about how Gödel's philosophy is related to Leibniz is found in [25]. There Gödel's biographer logician Hao Wang [25, p. 291] explains, based on conversations with Gödel, how Gödel's main line of philosophy aims to construct a neo-monadology that follows Leibniz. In the chronology of [24], there is a mention of Gödel's Leibniz studies as occurring from 1943 to 1946. These years are given by Gödel himself in the supplementary reply to a questionnaire printed in full in [24, pp. 16-21]. As we shall see below, this shows that Gödel had begun writing notes on the ontological proof prior to his systematical Leibniz studies. Though other reports (by Karl Menger) mention an earlier interest in Leibniz during the 1930's [24, pp. 48-49] or [25, p. 7]. This is also confirmed by the Nachlass, where Leibniz and Monadology are mentioned among notes on Quantum Mechanics written in $1935^{1}$.

\footnotetext{
${ }^{1}$ Box 6b, Folder 78, Reel 21, Frames from 967 and on.
} 
In the secondary literature on the ontological proof, the attribution of Gödel's proof to a study of Leibniz work is made primarily based on the plan of the formal argument from 1970 [23, p. 115] as well as the concepts used in this version (i.e. essence and positive properties vs. perfections). Leibniz is known for adding to the ontological argument a proof of the compatibility of perfections. Gödel's notes [11, Appendix B, pp. 434-435] contain a discussion of these two concepts, positive properties and perfections, as a possible interpretation of these. In [11, p. 389] some basic knowledge about Leibniz' comments on the ontological proof are explained and it is conjectured that Gödel knew about these texts, because they were widely accessible at that time, but little concrete evidence is given to support these claims.

The attribution of Gödel's proof to Leibniz is supported by the discussion between Wang and Gödel found in [24], but Wang is hesitant to address the topic of the ontological proof, because of cultural differences [24, p. 195] and [25, p. 129]. Nevertheless, Wang quotes Gödel, referring to this conversation as occurring in 1972, with respect to the ontological proof as saying that "he first got his idea of this proof in reading Leibniz" [25, p. 113]. However, this close connection to Leibniz is denied by Gödel in about 1977 when he is making corrections to Wang's manuscript [25, p. 87]; "I have never obtained anything definite on the basis of reading Leibniz. Some theological and philosophical results have just been suggested [by his work]. One example is my ontological proof [of the existence of God]. Dana Scott has [a copy of] the proof. It uses the division between positive and negative properties [proposed by Leibniz]." Though Gödel did not here attribute the ideas of his proof to anyone but Leibniz, there are other implicit influences.

In [24, p. 18], Gödel answers the above mentioned questionnaire about his professional background by referring to the philosophical introductory lectures by Heinrich Gomperz as an influence to which he attributes special significance in the development of his philosophy. The answer is confirmed in a supplementary reply that mentions only Gomperz' lectures as an important influence.

Some early notes ${ }^{2}$ in Gödel's Nachlass from Gomperz' lectures dated 'Winter 1925', and found on the same page as the title Gomperz and the subtitle History of European philosophy [Gomperz - Geschichte d. europ. Phil.], contain a list of names among others Augustinus, Anselm of Canterbury, Thomas Aquinas, William of Occam, as well as Meister Eckhart, some followed by approximate years or a short comment. Anselm's name has beneath it a note of 'ontological proof' [(Ontol. Gottesbew.)] but this particular list ends before any mention of Leibniz.

These particular 1925 notes of Gödel continue with some notes on the Philosophy of the Renaissance ${ }^{3}$ that focus on the relationship between belief and knowledge, which are indicated to equal the relationship between the belief systems of Plato and Aristotle [Verhältnis des Glaubens zum Wissen = Verhältnis der Glaubenssysteme zu Plato und Aristoteles]. The notes then culminate in

\footnotetext{
${ }^{2}$ Box 6b, Folder 72.5, Reel 21, Frame 503.

${ }^{3}$ This title is from Harald Höffding's Geschichte der neueren Philosophie Band 1. See Box 6b, Folder 72.5, Reel 21, Frames 581-582.
} 
a longer passage about Anselm of Canterbury where Gödel writes that Anselm aimed to derive his system of beliefs [... die Glaubenslehre beweisen] and that Anselm was convinced that the Church Doctrines, such as the Trinity, could be proved as faith by him was regarded the precursor of knowledge [Anselm von Canterbury ist überzeugt, dass alle Kirchenlehren, z.B. 3-Einigkeit, sich beweisen lassen. Um dieses Jahr 1200 - damals engster Zusammenhang zwischen Glaube und Wissen - credo ut intelligam. Glaube = Vorstufe des Wissens.]

These notes seem to indicate that Gödel had a broad and early interest in theology and the history of the ontological argument that can not be described as a pure interest in the formal logical axiomatization, which Gödel later expressed [1]. This claim is supported by the seemingly continuous production of theological notes made throughout his adult life. Gödel's motivation for the ontological proof is often related to the public opinion on Gödel's private beliefs (see also [24, p. 150, 194-195, 212] or [23, Chapter IV, section 1.2.] for a discussion of Gödel's motivation for the ontological proof), but a motivation for the argument can also be described as an interest in rational theology.

Other sources than Leibniz were studied by Gödel during the years when he developed his ontological proof. As is noted in [11, p. 390] the proof of Gödel "shows more affinity with a type of "ontological argument" based on modern modal logic'. In this context Charles Hartshorne's 1962 proof published in 'The Logic of Perfection' [15, pages 50-53] is mentioned by Adams as a potential source and indeed this can be verified by Gödel's notes. In the Nachlass ${ }^{4}$ there is an indisputable reference to Hartshorne's proof and that it has been studied. Gödel notes on a sheet of paper the source [Hartshorne über das ontol. Arg., Logic of Perfection $\overline{62}$, p. 50-51]. The reference is preceded by some formal notes related to Hartshorne's proof and followed by a few lines of notes in Gabelsberger. Evidently, this is another source that influenced the development of Gödel's ontological argument. Though, Hartshorne and his ontological argument are in [25, p. 146] severely, but broadly, criticised by Gödel on logical grounds.

As it is known that Gödel read Kant as early as 1922, it should also be taken into account that he certainly new Kant's objections against the ontological argument. For detailed information concerning Kant's objections, see for example [15, pp. 44] or [16, pp. 208].

\section{Gödel on propositions and principles of deduc- tion}

Much secondary work on Gödel's ontological proof centres around the development of an adequate formal system in which the ontological argument makes sense. Part of this approach is dealing with the potential modal collapse of this system. The modal collapse is defined by $A \rightarrow \square A$ being provable for all formulas $A$ in the system. The modal collapse was first pointed out in works by

\footnotetext{
${ }^{4}$ Box 10b, Folder 49, Reel 38, Frame 383-384.
} 
Sobel [22, p. 253] and [23, Ch. IV, sec. 6]. Subsequent development has taken various paths (1) emendations of the axioms (such as [2] and [7], or Hájek's [14] emendation that is based on Gödel's philosophical notes), (2) cautious comprehension principles [13], or (3) a distinction between intensional and extensional types where essence and positivity are interpreted extensionally (which implies rigidity) [9, Ch. 11, sec. 9] and [4]. The general outset seems to be that the philosophical implications of a modal collapse reduce the interest in the argument, but also Gödel's own contested intentions seem to matter (see for example [1, pp. 399-402], [9, Ch. 11, sec. 9] or [19]).

A consensus on Gödel's views and how to best interpret his wishes does not exist. A general agreement seems to exist on the formal level but that too only when all details are explicit enough to be computer analysed [6]. The standard proof of modal collapse relates to the definition of properties in the system, if the comprehension principle that generates properties is very inclusive, then the modal system collapses to a higher-order non-modal system. Some suggestions of 'natural' restrictions on properties that would exclude closed sentences have been proposed in [18]. However, this proposed restriction is apparently made only based on an analysis of Gödel's 1970-notes and Scott's version. As seen below, in section 7, there is clear evidence for a broad interpretation of what comprises a property in Gödel's intended sense. There, not only the God property is stated as being positive (Th. 1), but the existence of God as well as the necessary existence of God are stated as being positive properties (Th. 3). Also in the version given in section 6 there is a definition of $A(x)$, being in fact a closed sentence without free occurrences of $x$, which is assumed to be a positive property in (Th. 2). However, these kinds of properties completely disappear from Gödel's notes towards the 1970-version.

Other debates, spurred by solutions to the modal collapse $[9, \mathrm{Ch} .7$, sec. 3], include a debate of the behaviour of modalities in the context of quantifiers. The choice of constant or varying domains in the possible worlds corresponds to philosophical preferences, and in the formal setting whether to accept Barcan and converse Barcan formulas as allowed principles of deduction. In section 7 below, Gödel explicitly motivates equivalences in (Df. 4) by proving the converse Barcan formula $(N[(x) A(x)] \supset(y) N A(y))$. In the context of a symmetric accessibility relation, where the modal axiom $B$ holds, the converse Barcan formula will be equivalent to the Barcan formula itself [8, Sec. 7]. As KB is a sufficient system for versions of Gödel's ontological proof [17], this will be the case for modal systems of the proof even if they are weaker than S5. This seems to suggest that Gödel intended a constant domain interpretation.

It is clear from the notes of Gödel that he considered multiple general principles of modal reasoning, in relation to the ontological proof, and rejected for example a Barcan-like principle. On two numbered sheets of paper ${ }^{5}$, of which the first states that they should be filed under the papers concerning the ontological proof [kommt zu ontol. Bew], the second sheet contains a Barcan-like principle $N(\exists x) K(x) \supset(\exists x) N K(x)$. The formula is preceded by a question

\footnotetext{
${ }^{5}$ Box 10b, Folder 49, Reel 38, Frame 379-380.
} 
mark and followed by a note that it apparently is not valid [offenbar nicht].

As for another hot topic: the potential rigidity of names that was treated at length in [9, Ch. 9, sec. 2-3]. Rigidity means philosophically that the de dicto/de re distinction vanishes and formally that the de dicto use of modalities $N[\langle\lambda x . \varphi(x)\rangle(y)]$, which necessitates a sentence, is equivalent to the de re use of modalities $\langle\lambda x . N \varphi(x)\rangle(y)$, which ascribes a necessary property to an object. If we for a property write $\varphi$ for short, then the equivalence of the properties $N \varphi \equiv$ $\hat{x}[N \varphi(x)]$, stated as (Df. 3 ) in section 7 below, suggests that the definiendum is de dicto and the definiens is de re, corresponding to a rigidity condition exactly as given in [9, Def. 9.5.1].

However, note that this condition is used in the version of section 7 , for axiom 3: $P(\varphi)>P(N \varphi)$, which also occurs as axiom 2 in the earlier version of section 6 , but this axiom was changed for the 1970-version into $P(\varphi) \rightarrow N P(\varphi)$ (see also the discussion in section 7 ) which entails only the rigidity of positiveness [4, sec. 4]. Therefore, any particular claims about rigidity may apply only to the earlier versions of the ontological proof, given in this paper, but could have been rejected or refined for the later 1970-version. As a conclusion one may at least note, without anachronistic assumptions, that the issue of rigidity was to some extent considered by Gödel himself.

\section{Gödel on equality and being}

Secondary work on Gödel's ontological argument faces problems, not only because of Gödel's informal use of a logical system, but also the logical language has to be deduced from context. When it comes to the formal language of Gödel's ontological proof, equality is generally included because it occurs in an example of a positive property in Gödel's own 1970-version as well as in the Scott-version. The notion of equality in higher-order systems can be axiomatic by including it as primitive or defined through Leibniz' Law. By this definition,

$$
a=b \equiv_{\mathrm{Df}}(\forall \varphi)[\varphi(a) \leftrightarrow \varphi(b)]
$$

the equality notion reduces to equivalence between formulas. The definition can be described as stating replacement of equals in properties, in one direction, and in the other direction (which is more problematic) it states identity of indiscernibles. The latter direction, which stems from Leibniz' philosophy, is a convenient way of avoiding a primitive extensional equality.

In the Nachlass Gödel repeatedly returns to the interpretation of positive properties. In the notes [11, Appendix B, pp. 432-433], Gödel sketches axioms for the positive and negative properties. Noteworthy is axiom 4 which states that being is positive [Das Sein ist positiv]. This axiom can be formalized and is used in section 6 below of the ontological proof. The axiom can be seen as

a base case for an axiomatic generation of an inductive set. The positivity of being, formalized through the equality predicate, is no stranger to the argument. It occurs in Gödel's 1970 notes but there as a consequence of the replacement axiom for the positivity predicate based on necessary entailment. It also occurs 
as a provable property, from the same axiom as in Gödel's notes, in Scott's version of the ontological proof. There it is used to produce a contradiction in the proof that positive properties are possibly exemplified (Scott's Theorem 1 ). As was noted in [17, Section 5] the reliance on the equality predicate is not actually required in Scott's version. There is a proof without equality in the language, which is no surprise because Gödel's 1970-version does not rely on the equality predicate either, but only seems to reflect on equality as an explanation of the intended set of positive properties or as a reflection on how the positive properties correspond to the intended language. Clearly, Gödel intended for equality to play a role in his proof as a positive property.

In some other notes (see [11, pp. 434-435]), Gödel axiomatizes the positive properties as perfectives through: (1) A property is a perfective if and only if it implies no negation of a perfective, (2) the necessity of a perfective is a perfective, and (3) being is a perfective. This passage is continued in a footnote where Gödel states that it is enough to assume that there is a perfective, because being as a perfective then becomes provable. Then follows an interpretation of positives as assertions or tautologies with a comparison of the axiomatization. This passage is followed by a, much debated, note where Gödel motivates his preferred axiomatization. Gödel states that taking the formula

$$
P(\varphi) \rightarrow P(N \varphi)
$$

as an axiom (as is done in the sections 6 and 7 below) is an essential presupposition for the ontological proof. Taking the implication $\varphi(x) \supset N \varphi(x)$ as an assumption he calls the inferior way [der schlechte Weg], because the ontological proof then follows from compatibility of the positive properties or rather compatibility of any system of properties. These MaxPhil notes relate, by comparing the dates, to the version of section 7 (see the discussion in section 7 ). Note that these passages have motivated several emendations [3, p. 170] and [14, Section 3]. The former uses exactly this formula (1) as axiom (A6'), but interprets 'das Sein ist positiv' through essences. The latter emendation works in a system with full comprehension but avoids modal collapse through the modification of the axioms.

Gödel considers (see [11, Appendix B, pp. 432-433]) being a property that is even philosophically the cause of the world [Das Bejahen des Seins ist die Ursache der Welt], though the fundamental philosophical concept is cause [Der philosophische Grundbegriff ist die Ursache]. In the same passage, he equates properties with the cause of the discernibility of things [Eigenschaft $=$ Ursache der Verschiedenheit von Dingen]. This could be a philosophical motivation for a treatment of apartness through a Leibnizian definition.

Another note that occurs at least twice (though in slightly different form) in the Nachlass ${ }^{6}$ is the formal statement

$$
x \neq y>\exists \varphi \cdot[\varphi(x) \wedge \neg \varphi(y)]
$$

\footnotetext{
${ }^{6}$ Folder 10b, Box 49, Reel 38, Frames 379-380.
} 
This statement claims that apartness of objects necessarily implies discernibility. In one of the occurrences ${ }^{7}$ a third conjunct is added by which the property $\varphi$ is an essential property [ $\varphi$ ist eine ess. Eigenschaft]. However, this note is preceded by a question mark. In the other occurrence ${ }^{8}$ the formula is followed by a note that this property $\varphi$ does not need to be directly perceivable [Aber dieses $\varphi$ braucht nicht rein begrifflich zu sein.] This seems to indicate that he is considering this to be a classical existence and not a constructive one. As an intuitionistic basic concept, apartness may be preferred, as the concept was introduced in place of an equality relation by Brouwer, but classically treated, this implication (2) is equivalent to Leibniz' formula of identity of indiscernibles.

Therefore, it seems to be motivated to treat equality in its Leibniz formulation when it concerns Gödel's ontological proof. In appendix 10 we will, when giving the full formal proofs, use the basic calculus $\mathbf{N D}_{\mathbf{K}}$ from [17] that has exactly this feature.

\section{Axiomatizations in Gödel's Nachlass}

Just like the notes on theological history are scattered through the Nachlass there are more formal notes on the ontological proof than what is generally recognized. We will here mention three versions of Gödel's ontological proof that predate the known two page notes dated Feb 10, 1970 [11]. The 1970-version is a basis for the Scott-version of the proof [21], but the latter differs slightly from the version of Gödel. The main differences are that the positivity of God is taken as an axiom and that a missing conjunct from the definition of essence, stating that the essence-property is satisfied by the object, is added. The latter addition has been concluded to be vital for the consistency of the axiomatization [4], but this was unknown to Scott at the time (as stated in personal correspondence to the authors and C. Benzmüller). The former alteration of the argument was initiated by Scott who considered it a natural improvement of the axiomatization (see [4, sec. 2.3] for details concerning Scott's version).

However, the missing conjunct of the essence-property was not purely invented by Scott. As is commonly noted by scholars, the conjunct is missing in Gödel's 1970 notes, but does occur in the definition of essence found in Gödel's notes [11, Appendix B]. These notes were published in the Collected Works of Gödel but have not, to our knowledge, been treated before as a complete formal axiomatization of the ontological argument. Note however, that the concept of essence only appears in the third of these versions, conjectured to be the latest of these.

The axioms relevant for the versions of sections 6 and 8 have been deduced from context, and do not appear in connection to the stated theorems, but do appear elsewhere in Gödel's notes. Gödel uses multiple notations for positivity of properties $(P(\varphi)$ and $\varphi \in+)$ as well as multiple standard notations for implication. For the notation of intuitionistic implication Gödel uses the

\footnotetext{
${ }^{7}$ Folder 10b, Box 49, Reel 38, Frame 380.

${ }^{8}$ Folder 10b, Box 49, Reel 38, Frame 379.
} 
$\supset$, whereas the notation for classical implication is $\rightarrow$. Gödel also defines a necessary implication for which he uses the $>$ symbol.

\section{Version No. 1}

The $c a .1941$ version of the ontological proof occurs in Gödel's notes ${ }^{9}$ and has previously been transcribed in [11, Appendix B, p. 429]. The transcriptions differ in the formulas (Df. 3 and Th. 2), because in these notes Gödel has corrected some symbols by writing on top of previous notations while obviously forgetting to correct the notation elsewhere. Which notations are the intended ones has here been deduced from context. The axioms that are listed below and used in the proofs of the theorems are preceded by a star $\left(^{*}\right)$, because they do not occur in connection to these notes. The relevant axioms are standard axioms (Ax. 1) that also occur in the 1970-version, (Ax. 2) states that the 'necessity of a perfective is a perfective,' written in notes on the philosophy of the ontological proof, (Ax. 3) is equivalent to axiom 1 of Scott's version (both the uncontroversial, so called 'good direction,' and the controversial, 'bad direction,' of the implication), and (Ax. 4) is a formalization of 'the positivity of being'. The definitions include the second order predicate $\Phi, \Phi(\varphi)$ representing the necessary exemplification of the predicate $\varphi$.

\section{Axioms}

*Ax. 1: $P(\varphi) \cdot \varphi \supset_{N} \psi \rightarrow P(\psi)$

*Ax. 2: $P(\varphi) \rightarrow P(N \varphi)$

*Ax. 3: $P(\varphi) \leftrightarrow \sim P(\sim \varphi)$

*Ax. 4: $P(\hat{x}(x=x)) \quad$ "Das Sein ist positiv"

\section{Definitions}

Df. $1: \quad G \equiv \hat{x}[(\varphi)[\varphi \in+\rightarrow \varphi(x)]]$

Df. 2: $\Phi(\varphi) \equiv N(\exists x) \varphi(x)$

Df. 3: $A(x) \equiv(\exists \varphi)\left[(x)\left[\varphi(x) \equiv_{N} G(x)\right] . \Phi(\varphi)\right]$

\section{Theorems}

Th. 1: $G(x) \cdot \varphi \in+\rightarrow \varphi(x)$

Th. 2: $\quad A \in+\rightarrow \Phi(G)$

Th. 3: $\quad(\exists x) G(x) \rightarrow N(\exists x) G(x)$

${ }^{9}$ Box 12, Folder 41, Reel 46, Frame 333. 


\subsection{Proofs}

The proofs of the theorems can be informally sketched using linear Gödel-style deductions. The proof of (Th.2) uses the second order Barcan schema as an allowed principle of reasoning.

\section{Proof of Th.1}

1. $G(x) \rightarrow(\varphi)[\varphi \in+\rightarrow \varphi(x)] \quad$ Df.1

2. $G(x) \rightarrow \varphi \in+\rightarrow \varphi(x) \quad \forall$ Ex.

3. $G(x) \cdot \varphi \in+\rightarrow \varphi(x) \quad$ propositional reasoning

\section{Proof of Th.2}
4. $A \in+\rightarrow M(\exists x) A(x)$
as usual
5. $A \in+\rightarrow M(\exists x)(\exists \varphi)\left[(x)\left[\varphi(x) \equiv_{N} G(x)\right] . \Phi(\varphi)\right]$
Df. $A$
6. $A \in+\rightarrow M(\exists \varphi)\left[(x)\left[\varphi(x) \equiv_{N} G(x)\right] . \Phi(\varphi)\right]$
first order Th.
7. $A \in+\rightarrow(\exists \varphi) M\left[(x)\left[\varphi(x) \equiv_{N} G(x)\right] . \Phi(\varphi)\right]$
Barcan
8. $A \in+\rightarrow(\exists \varphi)\left[M(x)\left[\varphi(x) \equiv_{N} G(x)\right] \cdot M \Phi(\varphi)\right]$
$M(a . b) \rightarrow(M a . M b)$
9. $A \in+\rightarrow(\exists \varphi)\left[(x)\left[\varphi(x) \equiv{ }_{N} G(x)\right] . \Phi(\varphi)\right]$
Barcan, $M N a \rightarrow N a$

10. $A \in+\rightarrow \Phi(G)$

\section{Proof of Th.3}

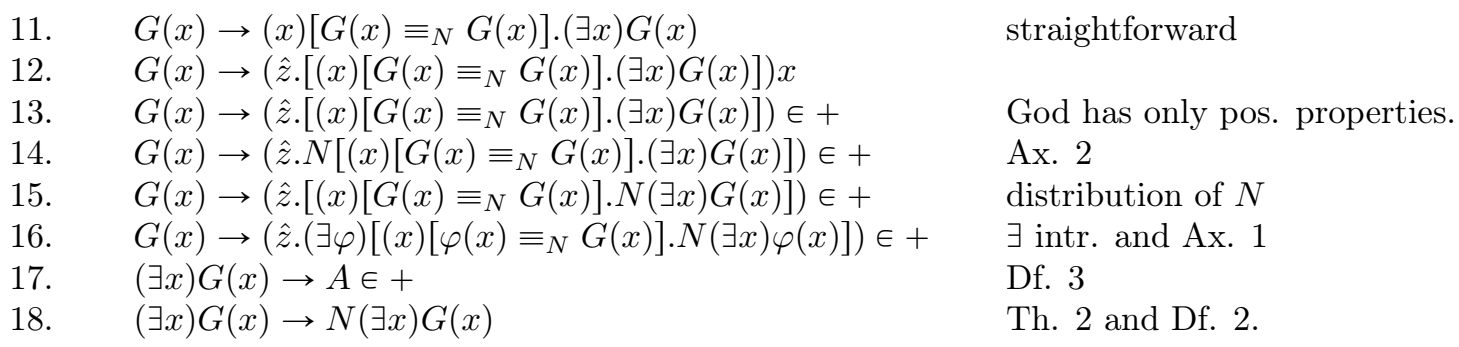

\section{Version No. 2}

The last page ${ }^{10}$ of this version has a note on the side of the page that dates it to 1952-53 [Siehe auch Heft über Max., Seaside Heights, ca. 52 oder 53]. This comment was apparently added later by Gödel. Compared to the Max. notebooks, MaxPhil 14 was written between 1946 and 1955, so this is the one in question. The comments about the ontological proof start at p. 103. On p. 101 it says "Asbury Park 1954," so the comments were written in (or after) 1954. This means that the MaxPhil14 comments relate to this 52/53-version. For example Gödel writes (p. 107) "Dass die Notwendigkeit einer pos. Eigenschaft pos. ist, ist die wesentliche Voraussetzung für den ontol. Bew." This axiom (stated below as Ax.3) was important for the first two versions occurring in this paper but changed for the 1970-version (into $P(\varphi) \rightarrow N P(\varphi)$ ).

\footnotetext{
${ }^{10}$ Box 12, Folder 41, Reel 46, Frame 336.
} 
In this version of the ontological proof, the axioms and theorems below occur as such in Gödel's Nachlass ${ }^{11}$. The definitions have, for a clear presentation, been reconstructed based on Gödel's axioms and theorems for this version. Note that $\Delta A$ denotes the first-order property of having all the properties sharing the second-order property $A$. The notes are clearly written and the theorems describe small steps of reasoning that are transparent. Therefore, no informal proofs are given, but the reader may consult the appendix of this paper for a formal derivation of each theorem.

\section{Axioms}

Ax 1. $\quad P(\varphi) \cdot \varphi>\psi .>P(\psi)$

Ax 2. $(\varphi)[A(\varphi) \supset P(\varphi)]>P(\Delta A) \quad$ Notw.

Ax 3. $P(\varphi)>P(N \varphi)$

Ax 4. $\quad P(\varphi) \supset \sim P(\sim \varphi)$

\section{Definitions}

Df 1. $\Phi(x) . \equiv .(\varphi)[P(\varphi) \supset \varphi(x)]$

Df 2. $\Delta A \equiv \hat{x}[(\psi)[A(\psi) \supset \psi(x)]]$

Df 3. $N \varphi \equiv \hat{x}[N \varphi(x)]$

Df 4. $\varphi>\psi . \equiv . N(x)[\varphi(x) \supset \psi(x)] . \equiv . N(x)[\varphi(x)>\psi(x)]$

da $N[(x) A(x) \supset A(y)]$,

$N[(x) A(x)] \supset N A(y)$

$$
\supset(y) N A(y)
$$

\section{Theorems}

Th $0 . \quad \varphi \equiv_{e} \psi \cdot P(\varphi) .>P(\psi)$

Th $1 . \quad P(\Phi)$

Th 2. $\Phi(x)>(\exists y) \Phi(y)$

Th 3. $\quad P(\hat{x}(\exists y) \Phi(y)), \quad N P[\hat{x} N(\exists y) \Phi(y)]$

Th 4. $\Phi(x)>(P[\hat{x} N(\exists y) \Phi(y)] \supset N(\exists y) \Phi(y))$

Th 5. $\Phi(x)>N(\exists y) \Phi(y)$

Th 6. $\quad(\exists y) \Phi(y)>N(\exists y) \Phi(y)$

Th 7. $\quad M(\exists y) \Phi(y)>N(\exists y) \Phi(y)$

Th 8. $\quad N(\exists y) \Phi(y)$

Th 9. $P(\varphi) \supset M(\exists x) \varphi(x)$,

da $\sim M(\exists x) \varphi(x) . \supset \varphi>\psi$,

da $N(x)[\varphi(x) \supset \psi(x)]$.

Also $P(\varphi) . \sim M(\exists x) \varphi(x) \supset(\psi) P(\psi)$.

It should be noted that the intended proof of the main Th. 8 can be significantly simplified. Instead of proving Th. 8 using Th. 9 note that it is possible to prove Th. 4 without the condition $\Phi(x)$. To this end, prove the right hand

\footnotetext{
${ }^{11}$ Box 12, Folder 41, Reel 46, Frames 335-336.
} 
side (conclusion) of Th. 4, using Th. 9 and $M N a \rightarrow N a$, which is valid in S5. Finally, detach Th. 8 by using Th. 3b.

\section{Version No. 3}

The date of this version is unknown. It has previously been transcribed in [11, Appendix B, p. 430], but here we treat it as a full axiomatization with some corrections in the transcription. The axioms have been added, but (Ax. 1) is referred to in Gödel's motivation for (Th. 1) and (Ax. 2) is a standard implication that follows from earlier stated definitions of God. The German notes on the righthand side of the formulas are Gödel's own.

It can be noted that the property essence and its definition does not occur in the versions above (sections 6 and 7). However, it does occur in this version below. Therefore, it seems that this concept was integrated into the ontological proof at a later stage perhaps as a consequence of his studies of Leibniz that occurred between 1943 and 1946.

In this proof the notation $E s s_{x}$ is used by Gödel both as a first order property and in the definition in his second footnote as a higher order property. However, by a standard proof any two essences of an object can be proved to be necessarily equivalent and thereby the essence of any object is unique. The use of essence as a predicate here can be compared to the 1970 version, which takes Ess as a binary relation between predicates and objects, and refines the definition of necessary existence to an implication exemplifying any essence of the given object.

\section{Axioms}

*Ax. 1: $\operatorname{Pos}(N E)$

*Ax. 2: $G(x) \supset(\varphi)(\operatorname{Pos}(\varphi) \supset \varphi(x))$

\section{Definitions}

Df. 1: $\quad G(x) \equiv_{D f} x$ ist Gott

Df. 2: $\quad N E(x) \equiv_{D f} N(\exists y) E s s_{x}(y) \quad$ notwendige Existenz

\section{Theorems}

Th. 1: $G(x) \supset N E(x)$

Th. $2^{12}: G(x) \supset . E s s_{x} \supset G \otimes$

Th. 3: $G(x) \supset N(\exists y) G(y)$

da $N E$ eine pos. Eigenschaft ist (gilt für jede Eigenschaft statt G)

(folgt aus den 3 Vorgehenden)

$(\exists x) G(x) \supset N(\exists y) G(y)$

$M(\exists x) G(x) \supset M N(\exists y) G(y)$

(beidseitiges Hinzufügen von $M$ )

daher

Th. 4: $\quad M(\exists x) G(x) \supset N(\exists y) G(y)$ 


\section{Gödel's Notes}

Ebenso folgt: Wenn der Begriff notwendige Existenz widerspruchsfrei ist, so gibt es Dinge, für die er gilt.

$\otimes$ Dazu braucht man, dass alle Eigenschaften Gottes durch eine Eigenschaft 2. Typs definiert sind. [Das muss überhaupt die Definition der Essenz sein.] Oder $E s s_{x}$ definiert durch: $\varphi \varepsilon E s s_{x} \equiv(\psi)\{\psi(x) \supset . N(x)[\varphi(x) \supset \psi(x)]\} \cdot \varphi(x)$

\subsection{Proofs}

\section{Proof of Th.1}

1. $G(x) \supset(\varphi)[\operatorname{Pos}(\varphi) \supset \varphi(x)] \quad$ Ax.2

2. $G(x) \supset \operatorname{Pos}(N E) \supset N E(x) \quad \forall$ Ex.

3. $G(x) \supset N E(x) \quad 2 . / \mathrm{Ax} .1$

Proof of Th.2 If $E s s_{x}$ is an essence of $x$ :

4. $(\psi)\left[\psi(x) \supset N(y)\left[\operatorname{Ess}_{x}(y) \supset \psi(y)\right]\right] \quad$ by Df.

5. $G(x) \supset N(y)\left[E s s_{x}(y) \supset G(y)\right] \quad \forall$ Ex., "Gilt für jede Eigenschaft statt $G$ "

6. $G(x) \supset\left(E s s_{x} \supset G\right) \quad$ abbreviation, overloading $\supset$

Note that Gödel uses $E s s_{x}$ both as a name for an essence and (in his second footnote) as a higher order predicate.

\section{Proof of Th.3}

7. $G(x) \supset N(\exists y) E s s_{x}(y)$

8. $G(x) \supset N(y)\left[E s s_{x}(y) \supset G(y)\right]$

9. $G(x) \supset N\left[(\exists y) E s s_{x}(y) \supset(\exists y) G(y)\right]$

10. $G(x) \supset N(\exists y) G(y)$
Th.1 / Df.2

Th. 2

8./ first order theorem: $\forall(A \rightarrow B) \rightarrow \exists A \rightarrow \exists B$

7./9.

Proof of Th.4 As usual.

\section{Conclusions}

Gödel's Nachlass offers multiple axiomatizations of the ontological argument as well as even further possible axiomatizations based on the philosophical notes. We have presented three complete versions of Gödel's ontological proof based on explicit notes in his Nachlass. Gödel's 1970 axiomatization has previously led to known emendations of the axioms that claim to better correspond to our intuitions about the defined notions and that do not lead to the modal collapse of the system. As we have shown, Gödel worked on the ontological proof during his whole adult life, and came into contact with it as early as 1925. The axiomatizations of Gödel show how he experimented with the formal

\footnotetext{
${ }^{12}$ The second $\supset$, written on top, was originally the symbol $=$. Probably Gödel's second footnote relates to the $=$-version.
} 
axioms, and weighed the potential axioms based on his philosophical preferences, touching upon several topics in the current debate on his proof. The 1970version can still be considered a final version, because Gödel expressed that he was satisfied with the proof (mentioned in Morgenstern's diary, see [1]), which was a work in progress during the writing of the earlier notes. However, the notes show how the proof developed formally based on a rough philosophical direction for the work.

\section{Appendix: Formal proofs in a natural deduc- tion system}

We will prove the theorems of each version of the ontological proof in the natural deduction calculus of [17]. The calculus is, as noted in the mentioned paper, an extension of a standard non-modal higher-order natural deduction calculus taken from [5]. The calculus can be regarded as a minimalistic calculus for a rigid higher-order modal logic $\mathbf{K}$ without extensionality principles. Stronger modal logics can be obtained by adding axioms/rules for specific systems (such as KB or S5). We will following Gödel use a classical S5 system. It can be noted that the calculus does not restrict the comprehension principle used, hence it has the flaw of having full comprehension that entails modal collapse, but with the benefit of being close to the language of theorem provers.

We will use below modal axiom 4: $\square A \rightarrow \square \square A$, which corresponds to transitivity in the model, and modal axiom $B^{*}: \diamond \square A \rightarrow A$ ( $B^{*}$ is sometimes referred to as Brouwer's reduction principle and can be derived from a standard formulation of axiom $B: A \rightarrow \square \diamond A$ ), which corresponds to symmetry in the model, as well as modal axiom $\mathrm{M}: \square A \rightarrow A$, which corresponds to reflexivity in the model. An additional reference is made to a point where a Barcan schema is used. This schema is, however, provable within our system, because we have constant domains, but it is pointed out in order to clarify that this is in fact an accepted way of reasoning that occurs in Gödel's notes.

A dotted line refers to an unfolding of definitions and double lines to shortened passages of already clear reasoning. The intuitionistic negation symbol

has been replaced with a classical $\neg$-symbol, the classical implication symbol $\rightarrow$ is used throughout the proofs, the $N$ for necessity and $M$ for possibility have been replaced by $\square$ and $\diamond$, and the dot-notation for conjunction has been replaced with a $\wedge$-symbol. All these notations follow the style of the given calculus. In addition the symbol $>$ is used for a necessitated implication.

\subsection{Variant 1 of the ontological proof}

Th. 1: 


$$
\begin{aligned}
& \frac{[\omega: G(x) \wedge \varphi \in+]^{1}}{\omega: G(x)} \wedge E \\
& \underset{\omega: \varphi \in+\rightarrow \varphi(x)}{\ldots \ldots \ldots(x) \wedge \varphi \in+]^{1}} \frac{[\omega: G(x)}{\omega: \varphi \in+} \rightarrow E \\
& \frac{\omega: \varphi(x)}{\omega: G(x) \wedge \varphi \in+\rightarrow \varphi(x)} \rightarrow I, 1
\end{aligned}
$$




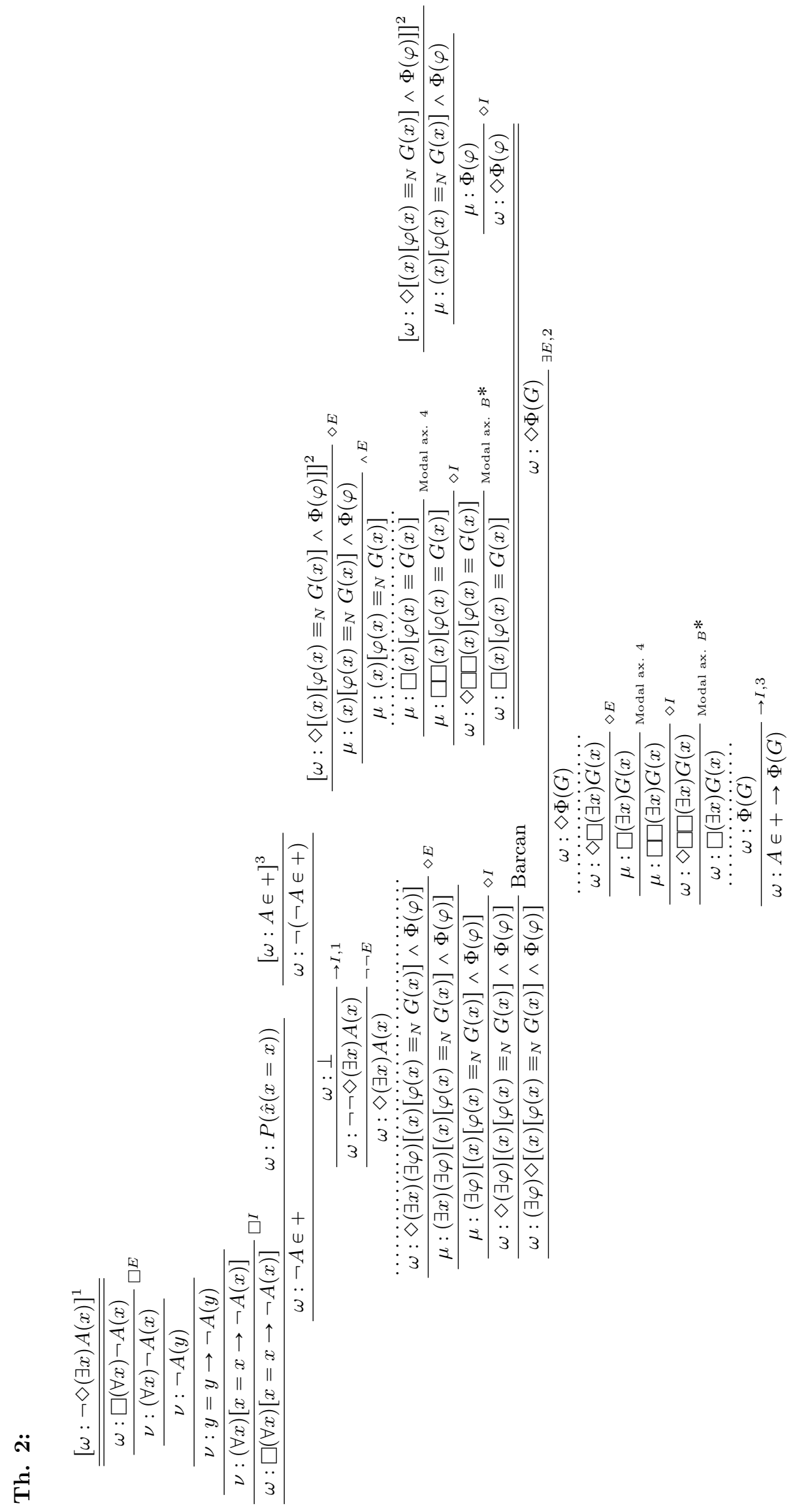


Th. 3:

$$
\begin{aligned}
& {[\omega: G(x)]^{1}} \\
& \omega:(\varphi)(P(\varphi) \rightarrow \varphi(x)) \text { Df. } 1
\end{aligned}
$$

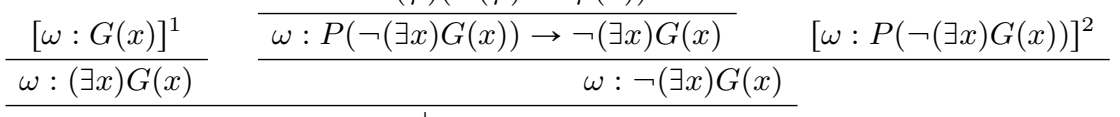

$$
\begin{aligned}
& \frac{\omega: \neg P(\neg(\exists x) G(x))}{\omega: P((\exists x) G(x))} \rightarrow I, 2 \\
& \overline{\omega:(x)\left[G(x) \equiv_{N} G(x)\right] \wedge(\exists x) G(x) \in+} \\
& \omega: \square\left[(x)\left[G(x) \equiv_{N} G(x)\right] \wedge(\exists x) G(x)\right] \in+ \\
& \overline{\bar{\omega} \omega:\left[(x)\left[G(x) \equiv_{N} G(x)\right] \wedge \square(\exists x) G(x)\right] \epsilon+} \\
& \omega:(\exists \varphi)\left[(x)\left[\varphi(x) \equiv_{N} G(x)\right] \wedge \square(\exists x) \varphi(x)\right] \in+ \\
& \omega: A \in+ \\
& \text { Df. } 3 \\
& \omega: \Phi(G) \ldots \text { Df. } 2 \\
& \omega: \square(\exists x) G(x)^{\text {Df. } 2} \rightarrow I \\
& \omega:(\exists x) G(x) \rightarrow \square(\exists x) G(x) \rightarrow I,
\end{aligned}
$$

\subsection{Variant 2 of the ontological proof}

Th. 0:

$$
\begin{aligned}
& \underline{\left[\omega: \varphi \equiv_{e} \psi \wedge P(\varphi)\right]^{1}} \\
& \frac{\omega: \varphi \equiv_{e} \psi}{\underline{\omega: \varphi>\psi}} \quad \quad \quad\left[\omega: \varphi \equiv_{e} \psi \wedge P(\varphi)\right]^{1} \quad \ldots \ldots \ldots \text { Ax } 1 . \\
& \omega: \varphi>\psi \\
& \begin{array}{ll}
\omega: \varphi>\psi \wedge P(\varphi) & \frac{\omega: \varphi>\psi \wedge P(\varphi) \rightarrow P(\psi)}{\omega: P(\psi)} \rightarrow E
\end{array} \\
& \frac{\omega: P(\psi)}{\frac{\omega: \varphi \equiv_{e} \psi \wedge P(\varphi) \rightarrow P(\psi)}{\omega: \varphi \equiv_{e} \psi \wedge P(\varphi)>P(\psi)}} \rightarrow I
\end{aligned}
$$

Th. 1:

Ax 2 .

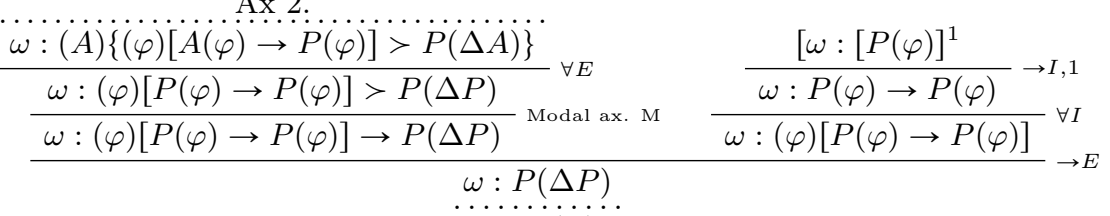

$$
\begin{aligned}
& \omega: P(\Phi)
\end{aligned}
$$

Th. 2:

$$
\frac{\frac{[\omega: \Phi(x)]^{1}}{\omega:(\exists y) \Phi(y)}}{\frac{\omega: \Phi(x) \rightarrow(\exists y) \Phi(y)}{\omega: \Phi(x)>(\exists y) \Phi(y)}} \rightarrow I
$$


Th. 3:

Th 2 .

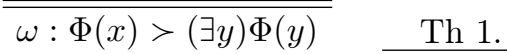

$$
\begin{aligned}
& \omega: \Phi>\hat{x}(\exists y) \Phi(y) \quad \frac{T}{\omega: P(\Phi)} \\
& \frac{\omega: \Phi>\hat{x}(\exists y) \Phi(y) \wedge P(\Phi)}{\omega: P(\hat{x}(\exists y) \Phi(y))} \text { Ax } 1
\end{aligned}
$$

Th. 3b:

$$
\begin{aligned}
& \text { Df } 3 . \\
& \frac{\omega:(\varphi)(\square \varphi \equiv \hat{x}[\square \varphi(x)])}{\omega: \square \hat{x}(\exists y) \Phi(y) \equiv \hat{x} \square(\exists y) \Phi(y)} \forall E \quad \frac{\overline{\omega: P(\hat{x}(\exists y) \Phi(y))}}{\omega: P(\square \hat{x}(\exists y) \Phi(y))} \text { Ax } 3 \\
& \frac{\omega: \square \hat{x}(\exists y) \Phi(y) \equiv \hat{x} \square(\exists y) \Phi(y) \wedge P(\square \hat{x}(\exists y) \Phi(y))}{\omega: P(\hat{x} \square(\exists y) \Phi(y))} \text {. } \\
& \frac{\omega: P(\hat{x} \square(\exists y) \Phi(y))}{\square P(\hat{x} \square(\exists y) \Phi(y))} \square I
\end{aligned}
$$

Th. 4:

$$
\begin{aligned}
& {[\omega: \Phi(x)]^{1}} \\
& \omega:(\varphi)[P(\varphi) \rightarrow \varphi(x)]
\end{aligned}
$$

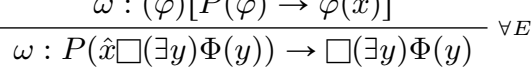

$$
\begin{aligned}
& \frac{\overline{\omega: \Phi(x) \rightarrow(P(\hat{x} \square(\exists y) \Phi(y)) \rightarrow \square(\exists y) \Phi(y))}}{\omega: \Phi(x)>(P(\hat{x} \square(\exists y) \Phi(y)) \rightarrow \square(\exists y) \Phi(y))} \square I
\end{aligned}
$$

Th. 5:

Th. 4.

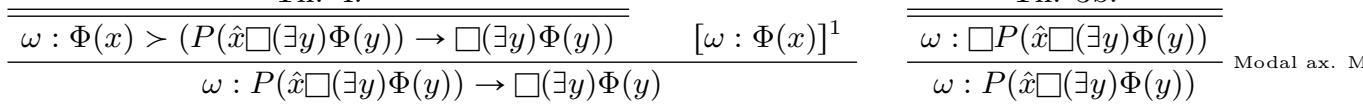

$$
\begin{aligned}
& \frac{\omega: \square(\exists y) \Phi(y))}{\omega: \Phi(x) \rightarrow \square(\exists y) \Phi(y)} \rightarrow I, 1
\end{aligned}
$$

Th. 6:

$$
\begin{aligned}
& \text { Th. } 5 . \\
& \frac{\overline{\omega: \Phi(x)>\square(\exists y) \Phi(y)}}{\omega: \Phi(x) \rightarrow \square(\exists y) \Phi(y)} \text { Modal ax. M } \\
& {[\omega:(\exists y) \Phi(y)]^{2}} \\
& \omega: \square(\exists y) \Phi(y) \\
& \omega: \square(\exists y) \Phi(y){ }_{\exists E, 1} \\
& \omega:(\exists y) \Phi(y) \rightarrow \square(\exists y) \Phi(y) \rightarrow I, \\
& \omega:(\exists y) \Phi(y)>\square(\exists y) \Phi(y)
\end{aligned}
$$

Th. 7: 
Th. 6 .

$$
\begin{aligned}
& \frac{[\omega: \diamond(\exists y) \Phi(y)]^{1}}{\nu:(\exists y) \Phi(y)} \diamond E \quad \frac{\overline{\frac{\omega:(\exists y) \Phi(y)>\square(\exists y) \Phi(y)}{\nu:(\exists y) \Phi(y) \rightarrow \square(\exists y) \Phi(y)}}}{\overline{ }} \square E \\
& \nu: \square(\exists y) \Phi(y) \\
& \nu: \square \square(\exists y) \Phi(y)
\end{aligned}
$$

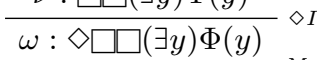

$$
\begin{aligned}
& \omega: \square(\exists y) \Phi(y) \\
& \frac{\overline{\omega: \diamond(\exists y) \Phi(y) \rightarrow \square(\exists y) \Phi(y)}}{\omega: \diamond(\exists y) \Phi(y)>\square(\exists y) \Phi(y)} \square I
\end{aligned}
$$

Th. 6.

Th. 8:

Th. 9

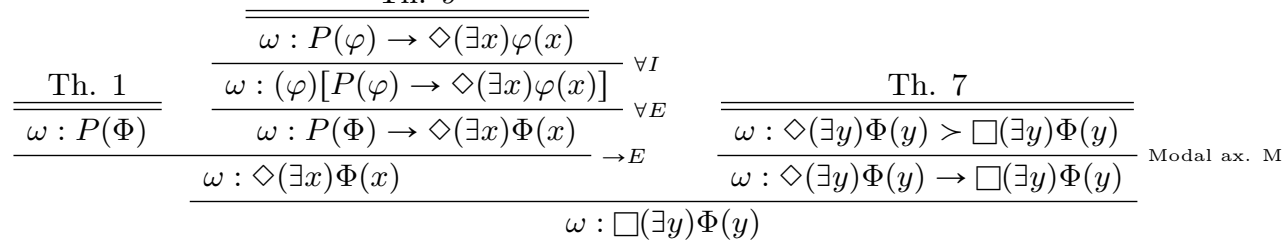

Th. 9:

$$
\begin{aligned}
& {[\omega: \neg \diamond(\exists x) \varphi(x)]^{2}} \\
& \omega: \square(x) \neg \varphi(x) \\
& \mu:(x) \neg \varphi(x) \\
& \frac{\mu: \neg \varphi(x)}{\mu: \perp} \quad[\mu: \varphi(x)]^{3} \\
& \frac{\mu: \perp}{\mu: \psi(x)} \perp E \\
& \mu: \varphi(x) \rightarrow \psi(x) \rightarrow I, 3 \\
& \mu:(x)[\varphi(x) \rightarrow \psi(x)] \\
& \omega: \square(x)[\varphi(x) \rightarrow \psi(x)] \\
& \begin{array}{rr}
{[\omega: P(\varphi)]^{1}} & \omega: \varphi>\psi \\
\hline
\end{array} \\
& \frac{\omega: P(\varphi) \wedge(\varphi>\psi)}{\omega: P(\psi)} \\
& \frac{\omega: P(\psi)}{\omega:(\psi) P(\psi)} \forall I \\
& \frac{\omega:(\psi) P(\psi)}{\omega: P(\neg \varphi)} \forall E
\end{aligned}
$$

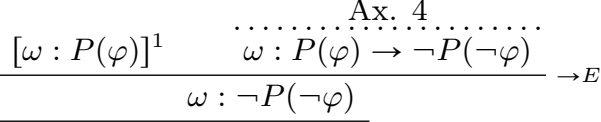

$$
\begin{aligned}
& \begin{array}{c}
\frac{\omega: \perp}{\omega: \neg \neg \diamond(\exists x) \varphi(x)} \rightarrow I, 2 \\
\frac{\omega: \diamond(\exists x) \varphi(x)}{\omega: P(\varphi) \rightarrow \diamond(\exists x) \varphi(x)} \rightarrow I, 1
\end{array}
\end{aligned}
$$

Note: Above we have followed Gödel's outline of how to prove the main Th. 8 relying on the subsequent Th. 9. See the end of section 7 for a hint of a shorter proof. 


\subsection{Variant 3 of the ontological proof}

Th. 1:

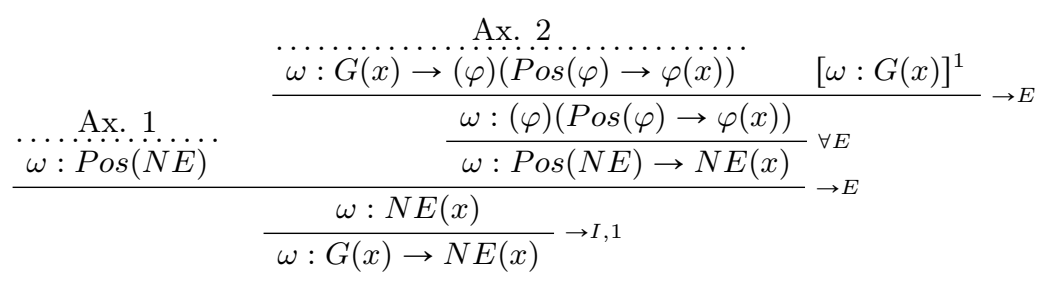

Th. 2:

Th. 1

$$
\begin{aligned}
& \frac{\frac{\omega: G(x) \rightarrow N E(x)}{\omega: N E(x)}[\omega: G(x)]^{1}}{[\omega} \\
& \ldots \ldots \ldots \\
& \omega: \square(\exists y) E s s_{x}(y) \text { Modal ax. M } \\
& \omega:(\exists y) E s s_{x}(y) \\
& \omega:(\exists \varphi)\left[\varphi \in E S S_{x} \wedge(\exists y) \varphi(y)\right] \\
& \omega: \varphi \in E S S_{x} \\
& \omega:(\psi)\{\psi(x) \rightarrow \square(y)[\varphi(y) \rightarrow \psi(y)]\} \wedge \varphi(x) \\
& \omega:(\psi)\{\psi(x) \rightarrow \square(y)[\varphi(y) \rightarrow \psi(y)]\} \rightarrow E \\
& \omega: G(x) \rightarrow \square(y)[\varphi(y) \rightarrow G(y)] \quad \forall E \quad[\omega: G(x)]^{1} \rightarrow E \\
& \omega: \square(y)[\varphi(y) \rightarrow G(y)] \\
& \overline{\omega: G(x) \rightarrow \square(y)[\varphi(y) \rightarrow G(y)]} \rightarrow I, 1 \\
& \omega: G(x) \rightarrow \square(y)\left(E s s_{x} \rightarrow G\right) \\
& {\left[\omega: G(x) \rightarrow(y)\left(E s s_{x} \rightarrow G\right)\right]}
\end{aligned}
$$

Th. 3:

Th. 2

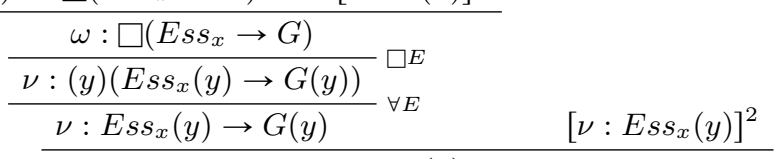

$$
\begin{aligned}
& \nu: G(y) \\
& \overline{\nu:(\exists y) G(y)} \exists E, 2 \\
& \frac{\nu:(\exists y) G(y)}{\omega: \square(\exists y) G(y)} \square I \\
& \omega: G(x) \rightarrow \square(\exists y) G(y) \rightarrow I,
\end{aligned}
$$

$\frac{\text { Th. } 1}{\omega: G(x) \rightarrow N E(x)} \quad[\omega: G(x)]^{1}$
$\ldots \omega: N E(x) \ldots \ldots$
$\frac{\omega: \square(\exists y) E s s_{x}(y)}{\nu:(\exists y) E s s_{x}(y)} \square E$

$\overline{\overline{\omega: G(x) \rightarrow \square\left(E s s_{x} \rightarrow G\right)}} \quad[\omega: G(x)]^{1}$

Th. 4: 


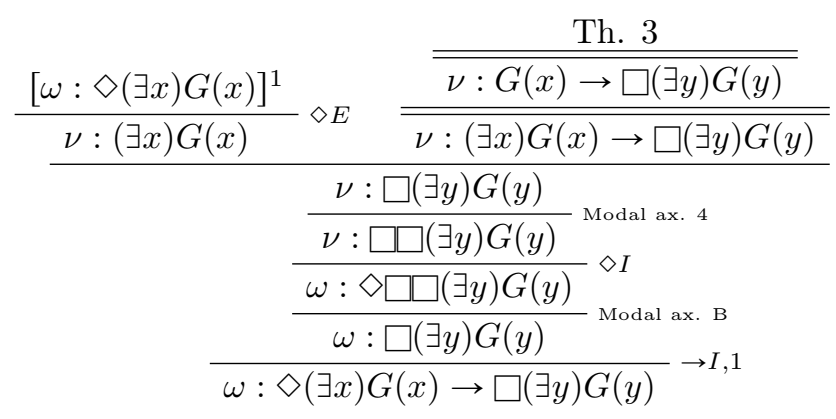

Acknowledgement: This project has received funding from the European Research Council (ERC) under the European Union's Horizon 2020 research and innovation programme (grant agreement No 787758).

\section{References}

[1] Adams, R. A. 1995. Introductory note to *1970, in: [11], Oxford University Press.

[2] Anderson, C. A. 1990. Some Emendations of Gödel's Ontological Proof. Faith and Philosophy, Vol. 7, Issue 3, pp. 291-303.

[3] Anderson, C. A. \& Gettings, M. 1996. Gödel's ontological proof revisited. In Hájek, P. (ed.), Gödel '96: Logical foundations of mathematics, computer science and physics-Kurt Gödel's legacy, Brno, Czech Republic, August 1996, proceedings, 167-172, Springer-Verlag, Berlin, 1996.

[4] Benzmüller, C. \& Fuenmayor, D. 2018. Can Computers Help to Sharpen our Understanding of Ontological Arguments? In Mathematics and Reality, Proceedings of the 11th All India Students' Conference on Science \& Spiritual Quest, 6-7 October, 2018, IIT Bhubaneswar, Bhubaneswar, India (Sudipto Gosh, Ramgopal Uppalari, K. Vasudeva Rao, Varun Agarwal, Sushant Sharma, eds.), The Bhaktivedanta Institute, Kolkata, www.binstitute.org, pp. 195-226.

[5] Benzmüller, C. \& Brown C. \& Kohlhase, M. 2004. Higher-Order Semantics and Extensionality. Journal of Symbolic Logic, Vol. 69, N. 4, pp. 1027-1088.

[6] Benzmüller, C. \& Weber, L. \& Woltzenlogel Paleo, B. 2016. ComputerAssisted Analysis of the Anderson-Hájek Ontological Controversy. In: Logica Universalis 10 (2016)

[7] Bjørdal, F. 1999. Understanding Gödel's Ontological Argument, in Timothy Childers (ed.) The Logica Yearbook 1998, pp. 214-217, Filosofia, Praha.

[8] Fitting, M. 1999. Barcan both ways, Journal of Applied Non-Classical Logics, Volume 9, Issue 2-3, pp. 329-344. 
[9] Fitting, M. 2002. Types, Tableaus, and Gödel's God, Kluwer Academic Publishers.

[10] Gödel, K. 1970. Ontological Proof, in Kurt Gödel Collected Works: Unpublished essays and lectures, Vol. 3, Oxford University Press, 1995.

[11] Gödel, K. 1995. Kurt Gödel Collected Works: Unpublished essays and lectures, Vol. 3, Oxford University Press, 1995.

[12] Gödel, K. 2003. Kurt Gödel Collected Works: Correspondence H-Z, Vol. 5, Eds. S. Feferman \& J. W. Dawson Jr. et al. Oxford University Press, 2003.

[13] Hájek, P. 1996. Magari and others on Gödel's Ontological Proof, in Ursini et al. (eds.) Logic and Algebra, pp. 125-136, Marcel Dekker.

[14] Hájek, P. 2002. A new small emendation of Gödel's ontological proof, Studia Logica (2002) Volume 71, Issue 2, pp. 149-164.

[15] Hartshorne, C. 1962. The Logic of Perfection, LaSalle, Il.: Open Court Publishing Company.

[16] Hartshorne, C. 1965. Anselm's Discovery - A Re-Examination of the Ontological Proof for God's Existence, LaSalle, Il.: Open Court Publishing Company.

[17] Kanckos, A. \& Woltzenlogel Paleo, B. 2017. Variants of Gödel's ontological proof in a Natural Deduction Calculus, Studia Logica, Vol. 105, Issue 3, pp. $553-586$.

[18] Koons, R. C. 2006. Sobel on Gödel's ontological proof, in Philosophia Christi, 8 (2006), 235-247.

[19] Kovač, S. 2012. Modal collapse in Gödel's ontological proof. In: Ontological Proofs Today, Chapter: 15. Publisher: Frankfurt etc.: Ontos, Editors: Mirosław Szatkowski, pp. 323-343.

[20] Muskens, R. 2006. Higher Order Modal Logic. In P. Blackburn, J.F.A.K. van Benthem, and F. Wolter, editors, Handbook of Modal Logic, Studies in Logic and Practical Reasoning, pages 621-653. Elsevier, Dordrecht, 2006.

[21] Scott, D. 1970. Notes in Dana Scott's hand. In: Sobel, J. H. 2001. Logic and Theism: Arguments for and against Beliefs in God, Cambridge University Press.

[22] Sobel, J. H. 1987. Gödel's Ontological Proof. In: edited by J. J. Thompson. On being and saying : essays for Richard Cartwright, MIT Press.

[23] Sobel, J. H. 2001. Logic and Theism: Arguments for and against Beliefs in God, Cambridge University Press.

[24] Wang, H. 1987. Reflections on Kurt Gödel, The MIT Press.

[25] Wang, H. 1996. A Logical Journey: From Gödel to Philosophy, The MIT Press. 